\title{
Considerations and guidance for using routine and program monitoring data for social and behavior change evaluation
}

Breakthrough RESEARCH

Follow this and additional works at: https://knowledgecommons.popcouncil.org/departments_sbsr-rh

Part of the Health Communication Commons, and the Public Health Commons

How does access to this work benefit you? Let us know!

\section{Recommended Citation}

Breakthrough RESEARCH. 2021. "Considerations and guidance for using routine and program monitoring data for social and behavior change evaluation," Programmatic Research Brief. Washington, DC:

Population Council. 


\section{Considerations and Guidance for Using Routine and Program Monitoring Data for Social and Behavior Change Evaluation}

Data collected routinely by governments and by program implementors can be leveraged to inform and evaluate social and behavior change (SBC) programs. What distinguishes routine data is that they are collected regularly within health information systems or within program monitoring systems. This brief is intended for global, regional, and country-level SBC program implementers, evaluators, and monitoring and evaluation teams who want to document whether their program is having an impact using routinely collected data. The brief provides an overview of the considerations of using routinely collected data for design and analysis, illustrates steps in undertaking an evaluation, and demonstrates how results can be applied to SBC programming.

\section{Introduction}

Understanding whether an intervention or activity is having its intended impact is considered program evaluation. Program evaluation can be defined as "a systematic method for collecting, analyzing, and using data to examine the effectiveness and efficiency of programs and, as importantly, to contribute to continuous program improvement." ${ }^{1}$ This brief will focus on the collection and analysis of routine and/or program data for evaluation in which the objective is to quantitatively assess the impact of a program or intervention, typically using statistical methods. To conduct program evaluation, data can come from primary or secondary sources.

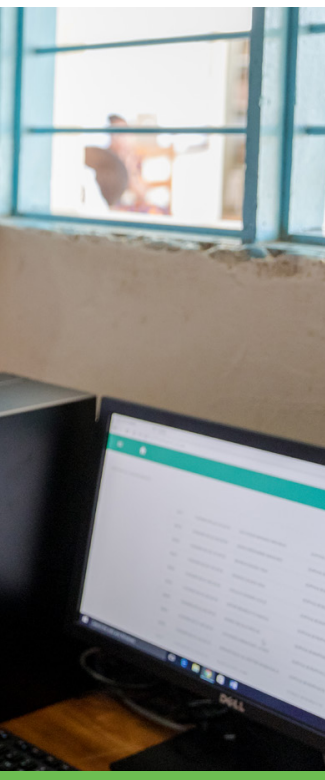

KEY POINTS

The use of routine and program monitoring data for evaluation presents many opportunities for evaluating the impact of programs on priority health outcomes.

There are several advantages for using routine and program monitoring data for evaluation, including that they are collected more frequently, across a wider geography, over a longer period of time, and may cost less to acquire.

Routine and program monitoring data may not be under the direct control of evaluators and thus may not always be appropriate for evaluation. Careful reflection on the appropriateness of the routine data for program evaluation is needed.

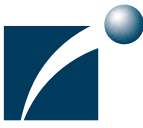


Primary data are collected for purposes of informing and evaluating a specific program or intervention, with the evaluators determining the indicators of interest to be collected and their measurement (how variables are defined, among what populations and geographies, and how often). The indicators may include intermediate outcomes of interest (knowledge, attitudes, norms, and practices), as well as behaviors and health outcomes, but may also include a wide array of potential measures that may impact the accuracy and precision of the empirical results (sociodemographics, other programming, or investments). The collection of primary data may not be possible for all evaluations due to program or research timelines and resources available for evaluation.

Secondary data refers to existing data sources in which an evaluator may have little or no control of their structure and/or collection. Routine data are considered secondary data. Routine data are collected on a regular basis by national governments and ministries within their information systems and are aggregated from sub-national units, e.g., health facilities, wards, districts, and other health administrative units. These data may be complemented by data collected routinely by an implementing program through its internal monitoring systems, e.g., collated from staff working in communities or program reports. Examples of such routinely collected data are noted in Table 1.

There are several advantages to using routine data over primary data for evaluation, including:

- Routine data are typically collected more frequently, across a wider geography, over a longer period of time.

- Routine data are less expensive for evaluators to obtain and potentially more rapidly available.

- As the data are typically not collected directly from individuals (human subjects) by the evaluators themselves, expedited review or exemptions by ethical review bodies may be possible and may facilitate data collection and analysis timelines.

However, as routine data may not be under the direct control of evaluators, they may not always be appropriate for evaluation. This brief outlines considerations for using routine data, and the design and analytical phases of program evaluation with routine data.

\section{TABLE 1 EXAMPLES OF POTENTIALLY AVAILABLE ROUTINELY COLLECTED DATA BY HEALTH AREA AND SOURCE}

\begin{tabular}{|c|c|c|c|}
\hline Health area & Data source & Data examples & $\begin{array}{l}\text { Recommended } \\
\text { frequency }\end{array}$ \\
\hline \multirow{3}{*}{$\begin{array}{l}\text { Family } \\
\text { planning } \\
\text { (FP) }\end{array}$} & Health facilities & Counts of individuals seeking FP services and adopting FP methods & Monthly \\
\hline & & Number of FP methods distributed to a health facility & \\
\hline & Program monitoring & $\begin{array}{l}\text { Counts of individuals attending community mobilization activities who } \\
\text { receive referrals for FP services }\end{array}$ & Monthly \\
\hline \multirow{5}{*}{$\begin{array}{l}\text { Maternal, } \\
\text { newborn } \\
\text { and child } \\
\text { health }\end{array}$} & Health facilities & Counts of pregnant women seeking antenatal care (ANC) services & Monthly \\
\hline & & Counts of pregnant women delivering at facility & \\
\hline & & Counts of immunized children & \\
\hline & Program monitoring & $\begin{array}{l}\text { Counts of pregnant women residing in households visited by program } \\
\text { who receive referrals for ANC services }\end{array}$ & Monthly \\
\hline & & Number of providers trained in respectful maternal and newborn care & \\
\hline \multirow{5}{*}{ Malaria } & Health facilities & $\begin{array}{l}\text { Counts of pregnant women taking intermittent preventive treatment } \\
\text { in pregnancy }\end{array}$ & Monthly \\
\hline & & Counts of individuals seeking malaria diagnostic and treatment services & \\
\hline & & $\begin{array}{l}\text { Number of rapid diagnostic tests and artemisinin-based combination } \\
\text { therapy administered }\end{array}$ & \\
\hline & Program monitoring & $\begin{array}{l}\text { Counts of individuals residing in households visited by program who } \\
\text { sleep under insecticide treated nets (ITNs) }\end{array}$ & Monthly \\
\hline & & Number of ITNs distributed during seasonal distribution campaigns & \\
\hline
\end{tabular}




\section{Considerations for using routine data for evaluation}

Before using routine data, evaluators need to ensure that these data are appropriate for the evaluation. Their appropriateness will be determined based on the specific research questions that have been developed, the resources available, and the evaluation timeline. Three considerations, and related questions to be answered, for using routine data for evaluation include:

- Usability:

$\checkmark$ Do the data include key indicators of interest that are expected to be changed in a meaningful way by the program, and can they be consistently measured over time?

$\checkmark$ Are the indicators available measured in a way that matches the program objectives, and is the measurement consistent over time?

$\checkmark$ Are the data collected at a level (e.g., individual, health facility, district) that is meaningful for the evaluation, and are there sufficient numbers of observations (across geographies and over time) at the level needed?

- Quality:

$\checkmark$ Are the data of sufficient quality to lead to valid and reliable results and conclusions, and does the quality of the data systematically vary over time or geographies?

$\checkmark$ Are the data sufficiently complete over time and across geographies to provide the necessary internal validity to draw conclusions about program impact?

- Accessibility:

$\checkmark$ Do the data require authorized approvals to obtain and use, and can the data be obtained and retrieved in time for the evaluation?

$\checkmark$ Does use of the data require review of results and conclusions by authorizing bodies who control the data?

If routine data are deemed to be appropriate for the evaluation, the next step is to develop the design of the evaluation.

\section{Designing an evaluation with routine data}

\section{Steps in designing an evaluation with routine data}

1. Collect key programmatic information and assess whether routine data are appropriate for evaluation

2. Develop an evaluation design

3. Develop a statistical analysis plan (SAP)

4. Establish data quality protocols

5. Create an integrated dataset for the evaluation

6. Conduct appropriate statistical analysis for routine data

\section{STEP Collect key programmatic informa- tion and assess whether routine data are appropriate for evaluation}

To design a rigorous and effective evaluation, it is important to have a clear understanding of the program's:

- Theory of change, which explains the pathways through which the program is expected to have an impact. It also indicates why it is expected that the program will ultimately have an impact on the indicators that are measured in routine data systems.

- Detailed implementation plans, which allow you to decide if comparison (counterfactual) areas are available, the timing of program initiation, phases of scale-up, and the geographies where the program will be implemented.

- Monitoring and tracking data that can be useful for the evaluation. The program monitoring data may provide useful quantitative information about program coverage, intensity, and resources used to implement the program.

\section{STEP Develop an evaluation design}

Evaluations can be experimental (includes randomized assignment of the program or parts of the program), quasi-experimental (has a non-randomized control group or 
has pre-/post-program measurement) or nonexperimental (no control group and a single post-program measurement). While randomized controlled trials are often considered the gold standard for evaluation, they are not always feasible for evaluating health interventions. Quasi-experimental designs that leverage routine data include, but are not limited to:

- Interrupted time series (ITS) that allows the evaluator to determine if there are shifts in the trend of an outcome indicator as a result of the program or a sudden interruption in programming (e.g., COVID-19). With ITS, the trend from the pre-program period is projected into the future and serves as the comparison (counterfactual) to the observed (actual) program trend.

- When program monitoring data are also available on reach (or exposure) and intensity of the program, a dose-response approach can be complementary to the ITS. Dose-response analyses help further assess the potential causal relationship between the program and outcomes.

Impact hypotheses: It is important in evaluation design to develop an a priori expectation, or hypothesis, as to the nature and magnitude of impact from the program, to distinguish it from an observed effect being found by chance, due to natural trends, or due to other factors unrelated to the program. This hypothesis may be informed by existing literature and/or the program's theory of change. For instance, when conducting ITS, a hypothesis would be formulated regarding the magnitude and trend of the outcome if the program were truly effective. For example, it may be expected that the program would cause an almost immediate change in the level of the indicator after initiation (Figure 1a) or, alternatively, a lag period with a more gradual change in the trend could be expected, as is illustrated in (Figure 1b). These hypotheses would inform the statistical evaluation of the impact.

For dose-response approaches, the impact hypothesis should specify the relationship expected between the intensity of the program (quantified) and the outcome (quantified). The location, timing, and intensity of program implementation is often collected in the program monitoring and tracking data, and can be paired with other routine data. Depending on the nature of the program and monitoring data collected, the intensity may be measured dichotomously (existence of a program/no program) or continuously, by the degree of program implementation (e.g., number of households visited in a village per month).

\section{STEP Develop a statistical analysis plan 3 (SAP)}

A prospectively formulated SAP increases the transparency and credibility of findings regarding program impact. It represents good scientific practice in program evaluation. An SAP should contain enough details so that the analysis can be replicated by others, and any changes in initial hypotheses and methods should be documented. Components of an SAP typically include $\mathrm{e}^{2-3}$ :

\section{FIGURE 1 HYPOTHETICAL PROGRAM IMPACT MODELS}

(a)

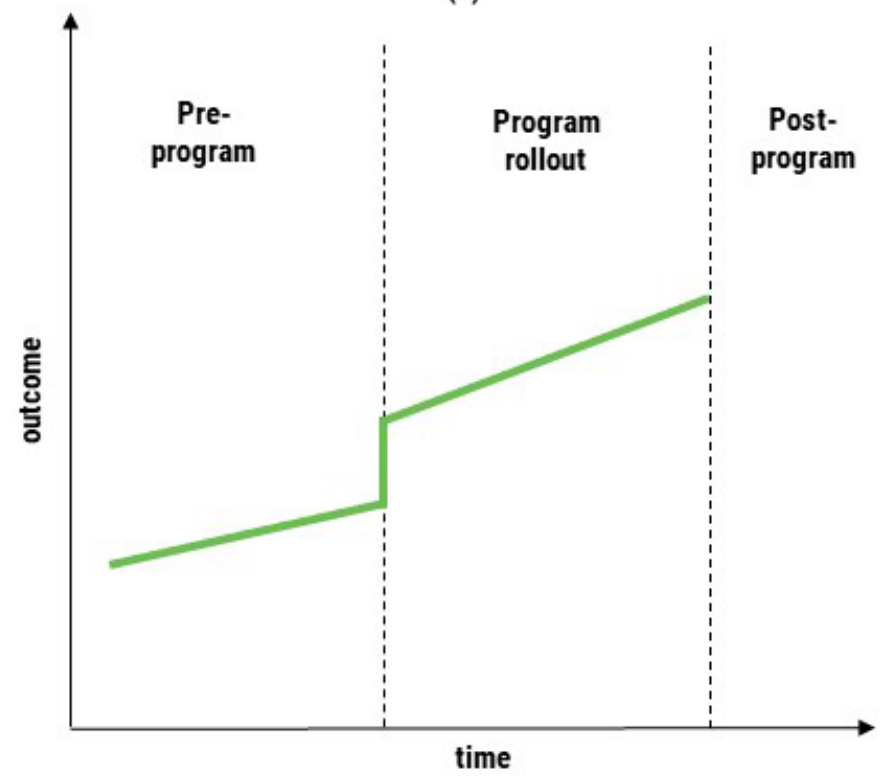

(b)

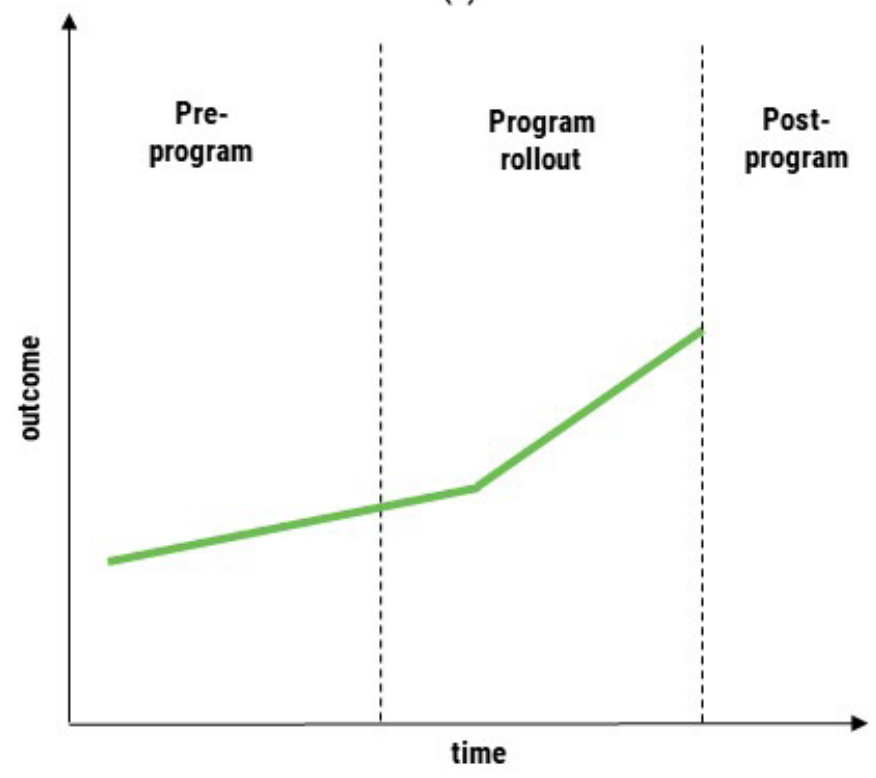


$\checkmark$ Administrative information: name of evaluation, current protocol version, SAP version, institutions, and principal investigators and roles.

$\checkmark$ The evaluation background, rationale, and objectives.

$\checkmark$ The evaluation methods: type of design, randomization (if applicable), statistical power considerations, null and alternative hypotheses, primary and secondary indicators, and timing of measurements.

$\checkmark$ Statistical principles: significance levels, expected effect sizes, adjustments for multiplicity hypothesis testing.

$\checkmark$ Study population: inclusion/exclusion criteria for data (e.g., based on quality), trends in indicators

$\checkmark$ Outcome definitions, measurement of treatment, confounding covariates

Statistical analysis methods.

\section{STEP Establish data quality protocols}

Data quality protocols describe what metrics and benchmarks will be used to assess the quality of the data. Examples of metrics, such as those developed by the World Health Organization, center around four dimensions of data quality relevant for routine data ${ }^{4}$ :

- Completeness and timeliness:

- Completeness of reporting: percent of expected data available at [level] over [time period].

- Timeliness of reporting: percent of expected data at [level] available on time.

- Completeness of indicator data: percent of data elements that are non-zero values and $\%$ of data elements that are non-missing values.

- Internal consistency:

- Outliers: percent of values that are above or below two standard deviations from the mean.

- Consistency over time: graphic depiction of trends.

- Consistency between related indicators: percent of cases where there are extreme differences between indicators that are expected to be roughly equal (e.g., first antenatal care visits and intermittent preventive therapy.

- External consistency: comparison of routine data with population-based survey values from similar period, if available.

- External consistency of population data used for denominators: for example, comparison of population data used by program with official government statistics.

\section{STEP Create an integrated dataset for the evaluation}

An evaluation using routine data will likely have to incorporate data from different sources for analysis. For example, routine data may be collected from health information systems, program monitoring data, and population data from censuses or other sources. Data from different sources should be carefully merged on matching identifiers, e.g., by time-period and geographical location and level. Documentation of the sources of data used to merge the data, their measurement, and how they were integrated is an important component of the SAP.

\section{STEP Conduct appropriate statistical analysis for routine data}

- Data analysis should commence with descriptive analysis (data frequencies, geographic information system (GIS) mapping, dashboards, and visualizations). When using routine data, scatter plots are a useful tool to identify underlying trends, seasonal patterns, and outliers. GIS mapping by geographic area is a powerful tool for visualizing large-scale program implementation.

- Interrupted time-series analysis: ITS regressions, also known as segmented regressions, can be estimated on a single group, where the pre-intervention trend projected into the treatment period serves as the counterfactual. ITS regressions can also be estimated to compare intervention and control groups. When using time series data, it is important to consider the following methodological challenges:

- Autocorrelation: consecutive observations tend to be more like one another than those further apart, which violates the assumption of standard regression models that observations are independent.

- Seasonality: indicators can have a seasonal pattern which can lead to biased results and autocorrelation.

- Time-varying confounders: ITS analysis ${ }^{\mathrm{a}}$ is generally unaffected by typical confounding variables that change relatively slowly over time, such as population age distribution or socioeconomic status;

aSee Lopez Bernal et al. $(2017)^{5}$ for a general tutorial on the use of ITS regressions for evaluation. 
denominator must be obtained from credible population data.

\section{Conclusion}

Evaluations of SBC programs using routinely collected health information and/or program data can be a useful tool for program evaluation. It is important to determine at the outset, when the evaluation and research questions are developed, whether routinely collected data are appropriate, specifically regarding the data's usability, quality, and accessibility. If the data are appropriate, such data has several advantages over primary data collection approaches. However, evaluations of routine data may not be feasible if health systems and/or program data are incomplete or of poor quality. Drawing together data from multiple sources into an integrated database may account for limitations and gaps in the data. To further improve upon the potential for using routinely collected data, it is preferable for evaluators to work with program implementers at the outset to ensure program data can be useful for program evaluation. ${ }^{11}$ Donors, implementers, and researchers are currently working hand-in-hand with national ministries in improving data collected in national information systems. 


\section{References}

1. U.S. Department of Health and Human Services Centers for Disease Control and Prevention. 2011. Office of the Director, Office of Strategy and Innovation. "Introduction to program evaluation for public health programs: A self-study guide." Atlanta, GA: Centers for Disease Control and Prevention.

2. Thomas, Laine, and Eric D. Peterson. 2012. "The value of statistical analysis plans in observational research defining high-quality research from the start," Journal of the American Medical Association 308(8): 773-774. doi: 10.1001/jama.2012.9502

3. Hiemstra, Bart et al. 2019. "DEBATE-statistical analysis plans for observational studies," BMC Medical Research Methodology 19: 233. doi: 10.1186/s12874-019-0879-5

4. World Health Organization. 2017. “Data quality review: a toolkit for facility data quality assessment-Module 1: Framework and metrics." Geneva: World Health Organization.

5. Lopez, Bernal James, Steven Cummins, and Antonio Gasparrini. 2017. "Interrupted time series regression for the evaluation of public health interventions: a tutorial," International Journal of Epidemiology 46(1): 348-355. doi: 10.1093/ije/dyw098

6. Turner, S.L. et al. 2020. "Design characteristics and statistical methods used in interrupted time series studies evaluating public health interventions: a review," Journal of Clinical Epidemiology 122: 1-11. doi: 10.1016/j.jclinepi.2020.02.006.

7. Linden, A. 2015. "Conducting interrupted time-series analysis for single- and multiple-group comparisons," Stata Journal 15: 480-500. doi: 10.1177/1536867X1501500208

8. ICF. "Demographic and Health Survey (DHS)" https://dhsprogram. com/Methodology/Survey-Types/DHS.cfm. [Accessed 1 September 2020].

9. Breakthrough ACTION. "Malaria behavior survey." https://malariabehaviorsurvey.org/ [Accessed 5 January 2020].

10. Breakthrough RESEARCH. 2020. "Twelve recommended SBC indicators for family planning," Breakthrough RESEARCH Programmatic Research Brief. Washington, DC: Population Council.

11. Centers for Disease Control and Prevention. 2019. "Program Performance and Evaluation Office," Webpage, 20 May 2019 [cited 8 October 2020]; Available from: https://www.cdc.gov/eval/index. htm.

\section{Suggested citation}

Breakthrough RESEARCH. 2021. "Considerations and guidance for using routine and program monitoring data for social behavior and change evaluation," Programmatic Research Brief. Washington, D.C.: Population Council.

Front cover photo credit: USAID

(C)2021 The Population Council. All rights reserved.
Email

BreakthroughResearch@popcouncil.org

\section{Breakthrough RESEARCH | Population Council}

4301 Connecticut Ave., NW, Suite 280 | Washington, DC 20008

+12022379400 | breakthroughactionandresearch.org

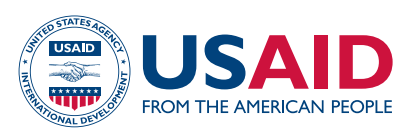

Breakthrough RESEARCH is made possible by the generous support of the American people through the United States Agency for International Development (USAID) under the terms of cooperative agreement no. AID-OAA-A-17-00018. The contents of this document are the sole responsibility of Breakthrough RESEARCH and Population Council and do not necessarily reflect the views of USAID or the United States Government.

\section{POPULATION} COUNCIL

Ideas. Evidence. Impact.

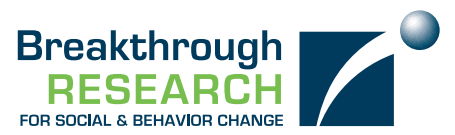

Breakthrough RESEARCH catalyzes social and behavior change (SBC) by conducting state-ofthe-art research and evaluation and promoting evidence-based solutions to improve health and development programs around the world. Breakthrough RESEARCH is a consortium led by the Population Council in partnership with Avenir Health, ideas42, Institute for Reproductive Health at Georgetown University, Population Reference Bureau, and Tulane University. 